PVP2006-ICPVT-11-93955

\title{
ENERGY TRANSFER BETWEEN AN ORBITING CYLINDER AND MOVING FLUID
}

\author{
László Baranyi \\ University of Miskolc \\ H-3515 Miskolc-Egyetemváros, Hungary \\ E-mail: arambl@uni-miskolc.hu
}

\begin{abstract}
This paper deals with the numerical simulation of low Reynolds number flow past a circular cylinder in orbital motion. Energy transfer between the incompressible fluid and a circular cylinder forced to follow an orbital path is investigated as a function of ellipticity. Limit cycles were investigated and show that ellipticity can have a large effect on the energy transfer. Sudden changes in state (jumps) were found when energy transfer is plotted against ellipticity. Phase angle was altered by about $180^{\circ}$ at the jumps. The effect of direction of orbit and initial conditions was also investigated.
\end{abstract}

\section{INTRODUCTION}

The near-wake structure of bluff bodies is extremely complex, and it seems that a theoretical approach cannot fully clarify this structure. For this purpose, either experimental or numerical analysis is needed. A huge amount of numerical and experimental work has been done on flow around a circular cylinder, centering on fixed cylinders, but also with a substantial number of studies on oscillating cylinders, primarily for transverse oscillation because of its higher applicability, but also for in-line oscillation. Sometimes the body moves in a combination of transverse and in-line oscillation, such as in tube bundles of heat exchangers. In this case a special combination of the two directions of oscillation is considered, when the resulting path of the cylinder is an ellipse. This can model, for instance, a cylinder affected by the motion of waves.

In oscillation studies, the standard aim is to investigate the fluid-structure interaction (FSI) of an elastically-supported body or structure placed in a moving flow. For FSI another method of numerical and experimental investigation is forced oscillation, where the body is mechanically moved. In all cases, that is in free or forced oscillation, and in transverse or inline oscillation, an important factor is the mechanical energy transfer that occurs between the fluid and the cylinder.
Oscillatory flow has been fairly widely researched (e.g. [1], [2]) as have oscillating cylinders in uniform flow (e.g. [3], [4], [5]). Also, in fluid at rest, an orbiting cylinder has been investigated numerically in [6], and in [7] authors investigated a cylinder that was orbiting and rotating. However, research on an orbiting cylinder in uniform flow seems to have been ignored.

For transverse forced oscillation there are several experimental (e.g. [8]) and numerical studies (e.g. [3] and [9]) where the amplitude of oscillation was fixed and the frequency of cylinder oscillation was varied in the vicinity of the natural vortex shedding frequency from a fixed cylinder at the same Reynolds number investigated. These studies found a sudden switch in streamline patterns within a very narrow frequency range, as well as a sharp change in the phase angle between the unsteady lift coefficient and the cylinder displacement. The authors in [3] demonstrated that the switch is associated with a change in the sign of energy transfer between cylinder and fluid and also with a sudden $180^{\circ}$ change in the phase angle between lift force and cylinder displacement. They attribute this switch to the competition between two vorticity production mechanisms, as it did not occur when just one was present. This paper describes a similar phenomenon for a more complex flow.

This study deals with an orbiting cylinder, in forced motion, placed in a uniform flow at low Reynolds numbers. Mechanical energy transfer, phase angle, and time-mean and root-mean-square values of lift, drag, and base pressure coefficients are investigated for quasi-periodic flow conditions for this as of yet undescribed phenomenon in orbiting cylinders.

One further factor rarely investigated is the influence of orbital direction (clockwise or anticlockwise). Studies of orbital cylinder motion tend to be carried out in only one direction. Initial condition, that is the point at which calculations are begun, may also have an effect on results. These factors are 
investigated here in order to confirm what effect, if any, they may have on energy transfer, phase angle or force coefficients.

\section{NOMENCLATURE}

$a_{0}$ cylinder acceleration, nondimensionalised by $U^{2} / d$

$A_{x, y}$ amplitude of oscillation in $x$ or $y$ directions, respectively, nondimensionalised by $d$

$C_{D}$ drag coefficient, $2 F_{D} /\left(\rho U^{2} d\right)$

$C_{L} \quad$ lift coefficient, $2 F_{L} /\left(\rho U^{2} d\right)$

$C_{p b}$ base pressure coefficient

d cylinder diameter

$D$ dilation

$e$ ellipticity, $A_{y} / A_{x}$

$E$ mechanical energy transfer, nondimensionalised by $\rho U^{2} d^{2} / 2$

$f \quad$ oscillation frequency, nondimensionalised by $U / d$

$\mathbf{F}$ force per unit length of cylinder, $F_{D} \mathbf{i}+F_{L} \mathbf{j}$

$F_{D} \quad$ drag per unit length of cylinder

$F_{L} \quad$ lift per unit length of cylinder

i, j unit vectors in $x, y$ directions, respectively

$p \quad$ pressure, nondimensionalised by $\rho U^{2}$

Re Reynolds number, $U d / v$

St nondimensional vortex shedding frequency

$t$ time, nondimensionalised by $d / U$

$T$ cycle period, nondimensionalised by $d / U$

$U$ free stream velocity, velocity scale

$u, v$ velocities in $x, y$ directions, nondimensionalised by $U$

$\mathbf{v}_{0} \quad$ cylinder velocity, nondimensionalised by $U$

$x, y \quad$ Cartesian co-ordinates, nondimensionalised by $d$

$\Phi$ phase angle

$v \quad$ kinematic viscosity

$\rho \quad$ fluid density

\section{Subscripts}

$L \quad$ lift

D drag

rms root-mean-square value

$x, y \quad$ components in $x$ and $y$ directions

1,2 for energy transfer in $y$ and $x$ directions, respectively

0 for cylinder motion; for stationary cylinder at same $R e$

\section{GOVERNING EQUATIONS}

A non-inertial system fixed to the cylinder is used to compute two-dimensional low-Reynolds number unsteady flow around a circular cylinder placed in a uniform stream and forced to follow an orbital path. The non-dimensional NavierStokes equations for incompressible constant-property Newtonian fluid, the equation of continuity and the Poisson equation for pressure are as follows:

$$
\frac{\partial u}{\partial t}+u \frac{\partial u}{\partial x}+v \frac{\partial u}{\partial y}=-\frac{\partial p}{\partial x}+\frac{1}{R e} \nabla^{2} u-a_{0 x}
$$

$$
\begin{gathered}
\frac{\partial v}{\partial t}+u \frac{\partial v}{\partial x}+v \frac{\partial v}{\partial y}=-\frac{\partial p}{\partial y}+\frac{1}{R e} \nabla^{2} v-a_{0 y} \\
D=\frac{\partial u}{\partial x}+\frac{\partial v}{\partial y} \\
\frac{\partial^{2} p}{\partial x^{2}}+\frac{\partial^{2} p}{\partial y^{2}}=2\left[\frac{\partial u}{\partial x} \frac{\partial v}{\partial y}-\frac{\partial u}{\partial y} \frac{\partial v}{\partial x}\right]-\frac{\partial D}{\partial t}
\end{gathered}
$$

In these equations, $u$ and $v$ are the $x$ and $y$ components of velocity, $t$ is time, $p$ is the pressure, $R e$ is the Reynolds number based on cylinder diameter, freestream velocity $U$, and kinematic viscosity, and $D$ is the dilation. Although $D$ is theoretically equal to 0 by continuity, it is kept in equation (4) for avoidance of the accumulation of numerical errors.

On the cylinder surface, no-slip boundary condition is used for the velocity and a Neumann type boundary condition is used for the pressure. At the far region, potential flow is assumed.

To be able to impose the boundary conditions precisely, a boundary-fitted coordinate system is used. The equations for connecting coordinate systems $(x, y, t)$ and $(\xi, \eta, \tau)$ are:

$$
\begin{aligned}
& x(\xi, \eta)=R(\eta) \cos [g(\xi)] \\
& y(\xi, \eta)=-R(\eta) \sin [g(\xi)] \\
& t=\tau
\end{aligned}
$$

where $\tau$ is the dimensionless time on the computational plane, and the dimensionless radius is

$$
R(\eta)=R_{1} \exp [f(\eta)]
$$

where the dimensionless cylinder radius is $R_{1}=0.5$. The structure of mapping automatically assures the orthogonality of the grid on the physical plane for arbitrary functions $f(\eta)$ and $g(\xi)$. A very fine grid can be obtained in the vicinity of the cylinder and a coarser grid far from the body, even with the linear functions for $f(\eta)$ and $g(\xi)$ used here (see Fig. 1). The equidistant mesh for the computational plane is beneficial for computational ease. The physical domain and the governing equations with boundary conditions are all transformed to the computational plane with an equidistant mesh, see [10]. The transformed equations are solved by finite difference method. Space derivatives are approximated by fourth order central differences, except for the convective terms for which a third order modified upwind scheme is used [11]. The Poisson equation for pressure is solved by the successive overrelaxation (SOR) method. The Navier-Stokes equations are integrated explicitly and continuity is satisfied at every time step.

The 2D code developed by the author has been extensively tested against experimental and computational results for fixed cylinders and good agreement has been found. The code was extended first for an oscillating and then for an orbiting cylinder. For this study the dimensionless time step was 0.0005 and the number of grid points was $301 \times 177$. For all $R e$ investigated, the solution was grid independent. The computational domain is characterised by $R_{2} / R_{1}=40$. 
It is proven that oscillation strengthens the twodimensionality of the flow (e.g. [12]) and for this reason a 2D code is suitable even at higher Reynolds numbers than the 190 at which three-dimensional effects start to appear for stationary cylinders [13].

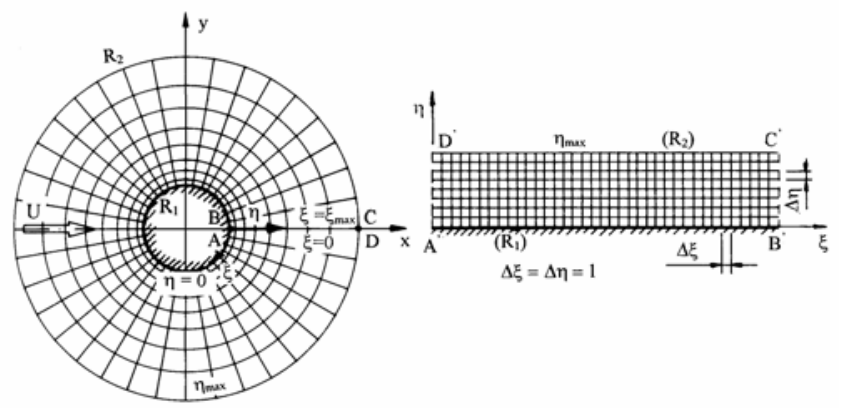

Figure 1 Mapping of the physical plane

\section{ORBITAL MOTION}

Figure 2 shows the flow arrangement for an orbiting cylinder. The orbital motion of the cylinder is created by the superposition of two forced oscillations with identical frequencies. The motion of the center of the cylinder with unit diameter is specified as follows:

$$
x_{0}(t)=A_{x} \cos \left(2 \pi f_{x} t\right) ; \quad y_{0}(t)=A_{y} \sin \left(2 \pi f_{y} t\right)
$$

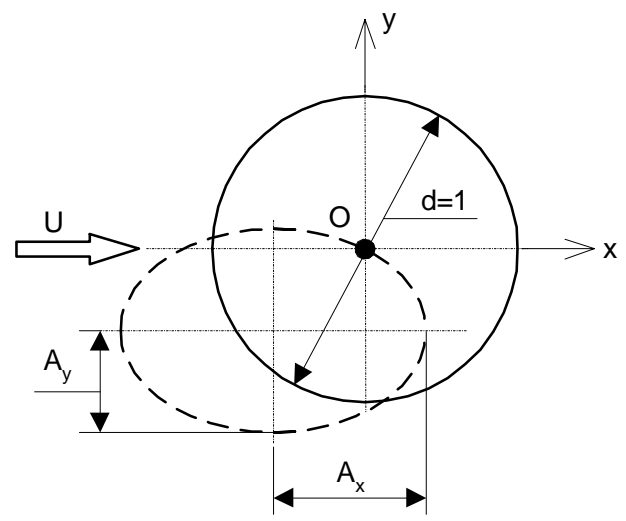

Figure 2 Orbital path of the cylinder

Here $f_{x}=f_{y}$ which for nonzero $A_{x}, A_{y}$ amplitudes gives an ellipse, shown in the dotted line in Fig. 2. $A_{x}$ alone yields pure in-line oscillation, and then as $A_{y}$ is increased, the ellipticity $e=A_{y} / A_{x}$ increases to yield a full circle at $e=1$. Equation (5) makes the cylinder orbit anticlockwise; by changing the sign of $y_{0}$ in equation (5) a clockwise orbit is obtained. Later in this paper, the effect of orbital direction and that of the initial condition are investigated.

During each set of computations, $R e$ and $A_{x}$ are fixed and $f_{x}$ and $f_{y}$ are kept constant at $90 \%$ of the frequency of vortex shedding from a stationary cylinder at that $R e$ (notated by $S t_{0}$ ).
This value was chosen to ensure lock-in (synchronisation of vortex shedding frequency with that of the cylinder oscillation) at moderate oscillation amplitudes.

An interesting phenomenon was observed when looking at the time-mean value (TMV; also denoted by overbar) and rootmean-square values of lift, drag and base pressure coefficients for an orbiting cylinder in a uniform flow. Abrupt jumps were found when these values were plotted against ellipticity $e$ with $R e$ and $A_{x}$ kept constant [14]. A typical example for the TMV of lift coefficient is shown in Fig. 3 (here $R e=120, A_{x}=0.4$, $f_{x}=f_{y}=0.85 S t_{0}$ ), where three sudden jumps in the curve can be seen. Both upper and lower curves are almost straight lines and in general their slopes are almost identical. Two different states were found on the curve of $\bar{C}_{L}$ versus $e$, one with greater lift, and the other with smaller. Both show an approximately linear decrease with increasing $e$, and the difference between the $\bar{C}_{L}$ values belonging to the two states is approximately constant. It was shown in [15] that the time histories of $C_{L}$ before and after the jumps are substantially different.

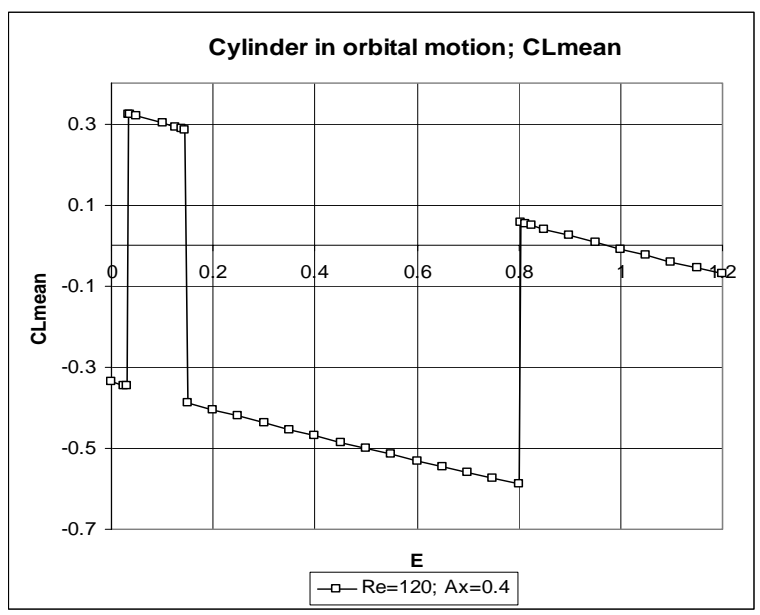

Figure 3 Time-mean value of lift vs. ellipticity

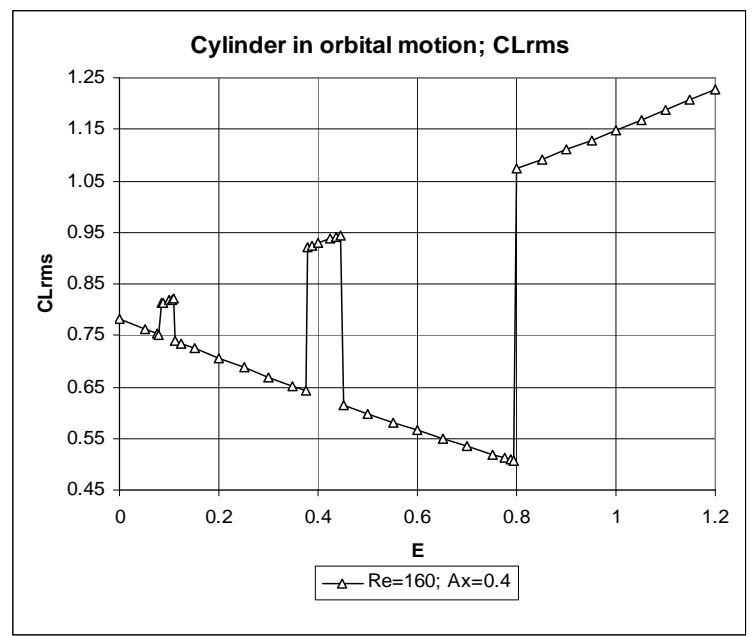

Figure 4 Rms value of lift vs. ellipticity 
The TMV and rms of drag and base pressure, further the rms of lift, behaved differently from $\bar{C}_{L}$, characterised by two envelope or state curves which are not parallel but intersect each other at $e=0$. A typical example is shown in Fig. 4. This finding also supports the idea that there are two states, or two solutions and the solution jumps from one state to the other and back. In this paper, the effect of these states on energy transfer is examined. Another concern is the investigation of phase angle versus ellipticity.

\section{ENERGY TRANSFER}

The mechanical energy transfer between fluid and a transversely oscillated cylinder was given in [3] and is here extended to encompass the bi-directional character of orbital motion. In this case, mechanical energy transfer $(E)$ takes place in both transverse and in-line directions. $E$ is determined when the flow is already quasi-periodic and hence $\left(y_{0}, C_{L}\right)$ and $\left(x_{0}, C_{D}\right)$ represent limit cycles. Energy transfer $E$ is positive when work is done on the cylinder, and negative when work is done on the fluid by the cylinder.

Extending Blackburn and Henderson's [3] definition of $E$ (mechanical energy transferred from the fluid to the moving cylinder per motion cycle), $E$ can be written as follows:

$$
\begin{aligned}
& E=\frac{2}{\rho U^{2} d^{2}} \int_{0}^{T} \mathbf{F} \cdot \mathbf{v}_{\mathbf{0}} d t=\frac{2}{\rho U^{2} d^{2}} \int_{0}^{T}\left(F_{D} v_{0 x}+F_{L} v_{0 y}\right) d t= \\
& \int_{0}^{T}\left(C_{D} \dot{x}_{0}+C_{L} \dot{y}_{0}\right) d t=E_{2}+E_{1}
\end{aligned}
$$

where $T$ is the motion period, $x_{0}, y_{0}$ means the dimensionless displacement of the cylinder in $x$ and $y$ directions, respectively, and a dot over these quantities means differentiation by dimensionless time. Naturally everything is dimensionless in the second line of equation (6). As can be seen, the energy transfer can be divided into two parts, $E_{1}$ and $E_{2}$. Using Green's theorem $E_{1}$ can be written for example as

$$
\begin{aligned}
E_{1}= & \int_{0}^{T} C_{L}(t) \dot{y}_{0}(t) d t=\oint C_{L} d y_{0}=-\oint y_{0} d C_{L}= \\
& \frac{1}{2}\left(\oint C_{L} d y_{0}-\oint y_{0} d C_{L}\right)
\end{aligned}
$$

Here line integrals are to be taken in clockwise direction. Similarly the energy transfer in the in-line direction is

$$
\begin{aligned}
E_{2}= & \int_{0}^{T} C_{D}(t) \dot{x}_{0}(t) d t=\oint C_{D} d x_{0}=-\oint x_{0} d C_{D}= \\
& \frac{1}{2}\left(\oint C_{D} d x_{0}-\oint x_{0} d C_{D}\right)
\end{aligned}
$$

The geometrical meaning of $E_{1}$ and $E_{2}$ is the signed area enclosed by limit cycles $\left(y_{0}, C_{L}\right)$ and $\left(x_{0}, C_{D}\right)$, respectively. They are positive when the orientation of the limit cycle curves is anticlockwise. Based on equation (6) the total energy transfer between fluid and cylinder is

$$
E=E_{2}+E_{1}
$$

\section{COMPUTATIONAL RESULTS AND DISCUSSION}

Computations were repeated for five different cases at 0.9 $S t_{0:}: A_{x}=0.4$ for $R e=120$ and $R e=140 ; A_{x}=0.2$ and $A_{x}=0.3$ for $R e=160$; and $A_{x}=0.3$ for $R e=180$. Here, results are given for just one case, as it generally represents all of the cases.

\section{ENERGY TRANSFER RESULTS}

Here, one set of results will be shown for the case of both clockwise and anticlockwise orbits at $R e=160, A_{x}=0.3$, and $f_{x}=f_{y}=0.9 S t_{0}=0.16938$ where $S t_{0}$ is the Strouhal number for a stationary cylinder at the given $R e$. Ellipticity $e$ was fixed for a single computation, then reset to a new $e$ value for the next, to cover regularly and fairly densely the ellipticity domain from 0 (pure in-line oscillation) to 1.2 (past a full circle). The reason for this was to be able to identify any jumps occurring in the domain. When a sign change occurred, several additional computations were performed on either side of the sign change. The initial condition for both orbital directions was set at $x_{0}(t=0)=A_{x}, y_{0}(t=0)=0$ (3 o'clock position). The data shown here is representative of the several sets of computations performed.

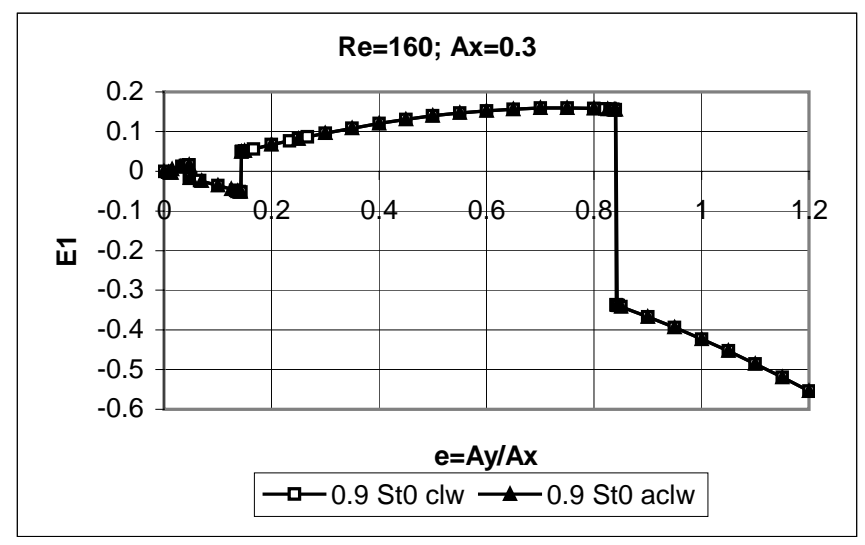

Figure 5 Transverse energy transfer $E_{1}$ vs. ellipticity

Figures 5-7 show the mechanical energy variation with ellipticity for the parameters given above, for both clockwise and anticlockwise directions of orbit. Figure 5 gives energy transfer between the fluid and the body in the transverse direction $E_{1}$. The empty squares show data for the clockwise direction (clw in the figure), and the filled triangles show data for the anticlockwise direction (aclw). The location of the jumps also seems to coincide, with the possible exception of one jump very near $e=0$, which needs to be examined more carefully. Each orbital direction produces one pair of envelope curves, which intersect each other at $e=0$, and these two pairs 
of envelope curves coincide with each other. Note that $E_{1}$ values are positive for the upper envelope curve, meaning that energy is transferred from the fluid into the cylinder, and negative for the lower curve, with the energy transfer reversed, acting to dampen the motion.

Figure 6 shows the energy transfer in in-line direction $E_{2}$ versus $e$. Many features of the figure are similar to those of Fig. 5 (two pairs of coinciding envelope curves), although here all of the $E_{2}$ values are negative for both the upper and lower envelope curves. This means that the force acting on the cylinder from the fluid would oppose the cylinder motion if it weren't mechanically forced motion. Compared with Fig. 5, the jumps occur at basically the same values of $e$, and the shape of the envelope curves is more or less reversed.

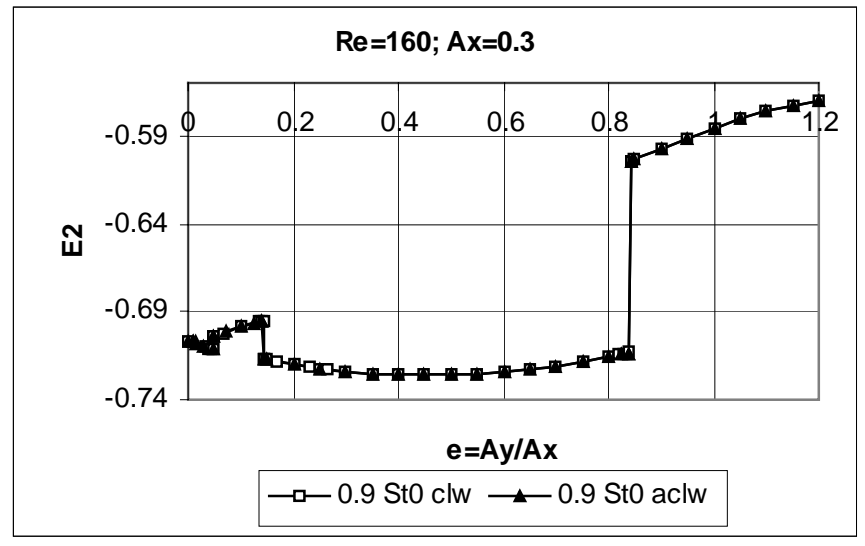

Figure 6 In-line energy transfer $E_{2}$ vs. ellipticity

The sum of the energy transfer in transverse and in-line directions is given in Fig. 7. The shape is that of $E_{1}$ but, like $E_{2}$, all of the values are negative. Therefore, the energy transfer for an orbiting cylinder was found to be always negative, regardless of direction of orbit, in the ellipticity domain investigated. The work, therefore, is done on the fluid by the cylinder, and naturally the fluid produces a kind of resistance against the forced motion of the cylinder.

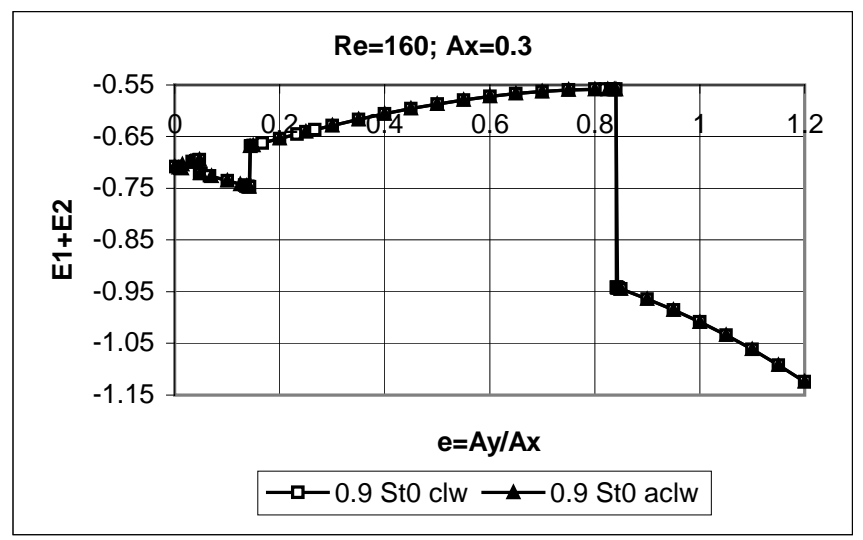

Figure 7 Total energy transfer $E$ vs. ellipticity
Next we will take a closer look at the jumps. This has been done by investigating the time-history lift coefficient curves [15], where it was found that the shape of the signals before and after a jump were substantially different, leading to different TMV and rms lift values. Here, the limit cycles are investigated before and after a jump, for a clockwise orbit at the parameters given at the beginning of this subsection. The jump investigated was at about $e=0.1435$ (see, for instance, Fig. $5)$. Figure 8 shows the two limit cycles $\left(y_{0}, C_{L}\right)$ in transverse direction for quasi-periodic motion. The limit cycle for the $e$ value before the jump, at $A_{y}=0.0429(e=0.143)$, is shown by the thinner line. The thicker line represents the limit cycle just after the jump, at $A_{y}=0.0432(e=0.144)$. Although the e-values hardly differ, the two limit cycle curves are completely different, almost reflecting each other, and with a change of orientation of traverse around the limit cycles. This means that the sign of energy transfer is opposite for the two curves: negative for $e=0.143\left(E_{1}=-0.0521\right.$ ) and positive for $e=0.144$ ( $E_{1}=0.0491$ ). This finding is very similar to that of [3] and [16] for a transversely oscillated cylinder with varying oscillation frequency. They also found a change in the orientation of the limit cycles, indicating a sign change in energy transfer.

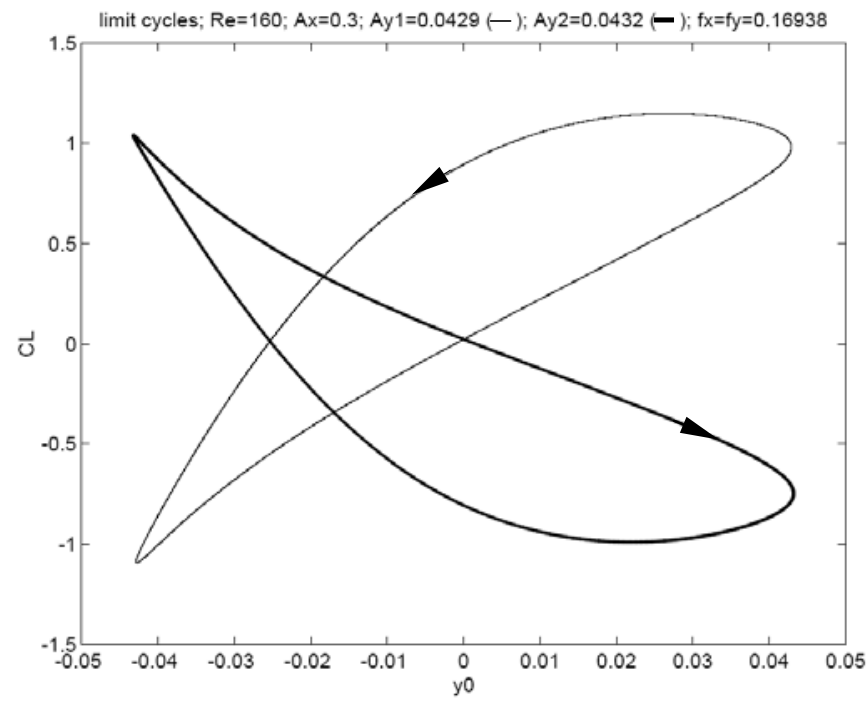

Figure 8 Limit cycles $\left(y_{0}, C_{L}\right)$ for $\boldsymbol{R e}=160, A_{x}=\mathbf{0 . 4}$, (thin line $-A_{y}=0.0429$; thick line $-A_{y}=0.0432$ )

Figure 9 shows the two limit cycles $\left(x_{0}, C_{D}\right)$ in in-line direction for quasi-periodic motion. Again the limit cycle for the $e$ value before the jump, at $A_{y}=0.0429(e=0.143)$, is shown by the thinner line. The thicker line represents the limit cycle just after the jump, at $A_{y}=0.0432(e=0.144)$. As can be seen in this figure, in contrast with the results shown in Fig. 8, the small change in the value of ellipticity did not cause any drastic change in the two limit cycle curves. The shape of the curves is almost the same and their orientation is identical. This orientation means negative energy transfer values: $E_{2}=-0.6947$ for $e=0.143$ and $E_{2}=-0.7663$ for $e=0.144$. As can be seen, the 
absolute value of $E_{2}$ is much larger than that of $E_{1}$ with the same $e$ values, so that the overall energy transfer $E$, i.e. the sum of $E_{1}$ and $E_{2}$, is negative for both of these cases.

Interestingly, the limit cycle for transverse displacement changes radically with a tiny change in ellipticity, but the limit cycle for in-line motion hardly changes at all. This means that the limit cycle for the lift coefficient and nondimensional transverse displacement is much more sensitive to the phenomenon causing the jumps than is the limit cycle based on the drag coefficient and in-line displacement. This suggests that lift and drag will behave differently in other measures of examining the jump phenomenon, such as phase angle analysis.

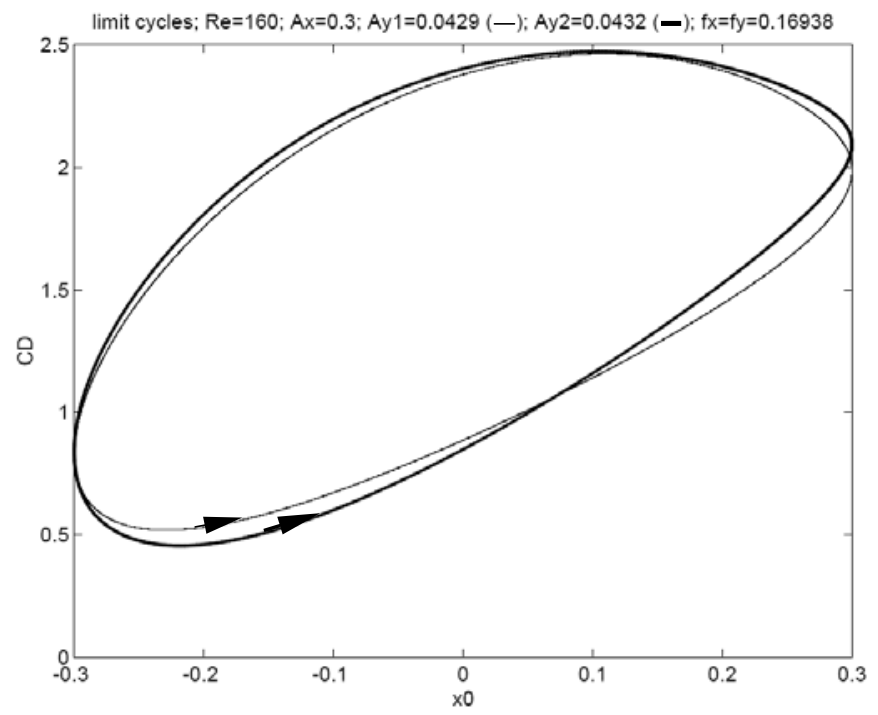

Figure 9 Limit cycles $\left(x_{0}, C_{D}\right)$ for $\mathbf{R e}=160, A_{x}=0.4$

(thin line $-A_{y}=0.0429$; thick line $-A_{y}=0.0432$ )

\section{PHASE ANGLE RESULTS}

Several studies ([3], [9], [16]) have shown a sudden shift occurring in the phase angle under lock-in condition when the cylinder oscillation frequency is in the vicinity of the vortex shedding frequency from a stationary cylinder at the same Reynolds number. Because of this, it seemed worthwhile to investigate the phase angle $\Phi_{L}$ between the lift coefficient and displacement of the cylinder in transverse direction.

Figure 10 shows that there is indeed a drastic change in the phase angle $\Phi_{L}$. The solid line, the one with a sine wave with the amplitude of 1.5, represents the time-history of displacement. The amplitude, which is considerably smaller in reality, has been exaggerated here to provide a convenient means to visualise the phase angle. The dotted line is the lift coefficient at $e=0.0429$, prior to the jump, while the heaviest line (composed of + signs) is the lift at $e=0.0432$, after the jump. The pre-jump curve is basically in phase with the cylinder displacement, while the post-jump curve is essentially reversed, yielding a phase shift of about $180^{\circ}$. So the jump has created a sudden change in the phase angle of about $180^{\circ}$ for lift.
The effect of the jump on the phase angle between drag and in-line cylinder displacement, on the other hand, is almost negligible, as seen in Fig. 11. The solid line again represents the time-history of displacement. The two hardly distinguishable lines are the pre-jump drag coefficient at $e=0.0429$ (dotted line), and is the post-jump drag at $e=0.0432$ (the heaviest line).

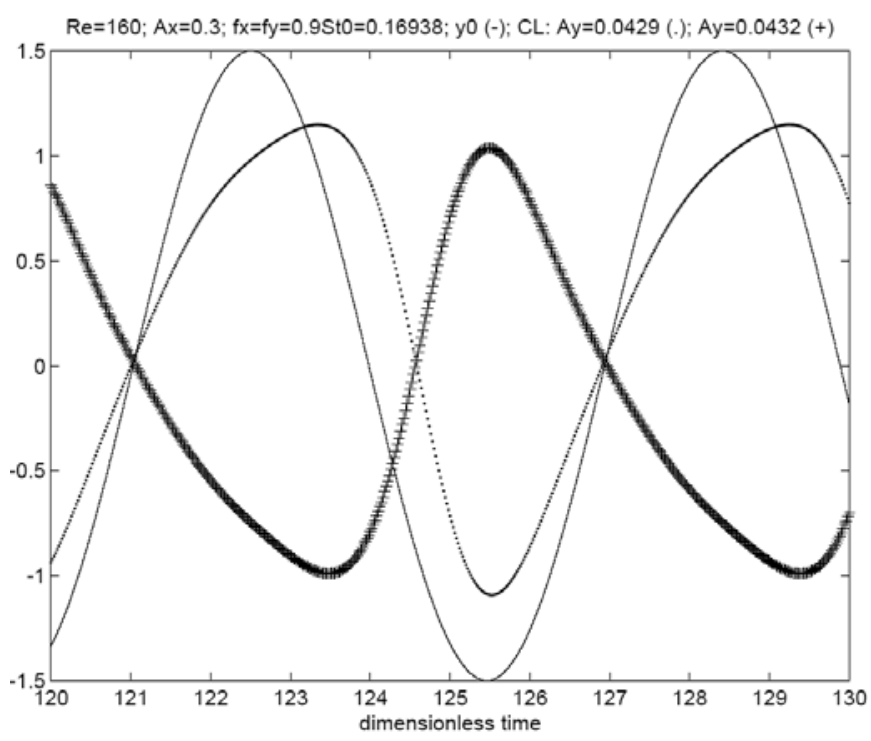

Figure 10 Time history lift and cylinder displacement curves (solid line - transverse cylinder displacement; dotted line $C_{L}$ at $A_{y}=0.0429 ;+$ line $-C_{L}$ at $A_{y}=0.0432$ )

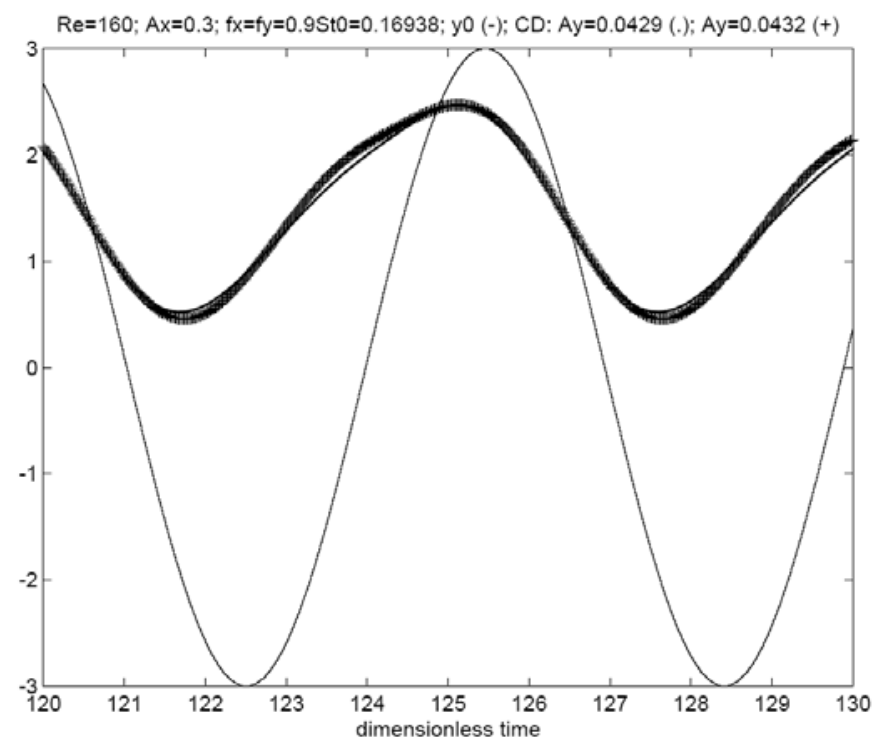

Figure 11 Time history drag and cylinder displacement curves (solid line -in-line cylinder displacement; dotted line - $C_{D}$ at $A_{y}=0.0429 ;+$ line $-C_{D}$ at $A_{y}=\mathbf{0 . 0 4 3 2}$ )

These findings suggest that any follow-up investigation should focus on phase angle between lift and transverse displacement. Therefore, for the representative case, $\Phi_{L}$ is 
determined and plotted versus ellipticity. This is shown in Fig. 12. This curve identifies the same locations for jumps as the other curves shown earlier (Figs. 5-7). Phase shift through the jumps is about $180^{\circ}$, which is in agreement with the findings of [3], [9] and [16].

One hypothesis for the sudden changes in phase angle in the case of a transversely oscillated cylinder is that the change in flow structure leading to shifts results form a change in balance between two different vorticity production mechanisms [3], [16]. This hypothesis is largely supported by the evidence gained in these two studies, and it seems a likely explanation for the phenomenon found here for orbiting cylinders. Figures 13a and 13b display a flow visualisation based on the numerical data, showing near-wake streamlines at a time of maximum cylinder displacement for the two earlier investigated ellipticity values (pre-jump and post-jump, for the same jump as earlier investigations). These two figures illustrate the fact that the timing of vortex shedding is changed dramatically by a very small change in the ellipticity (from $e=0.143$ to $e=0.144$ ). These results are similar to those obtained in [16].

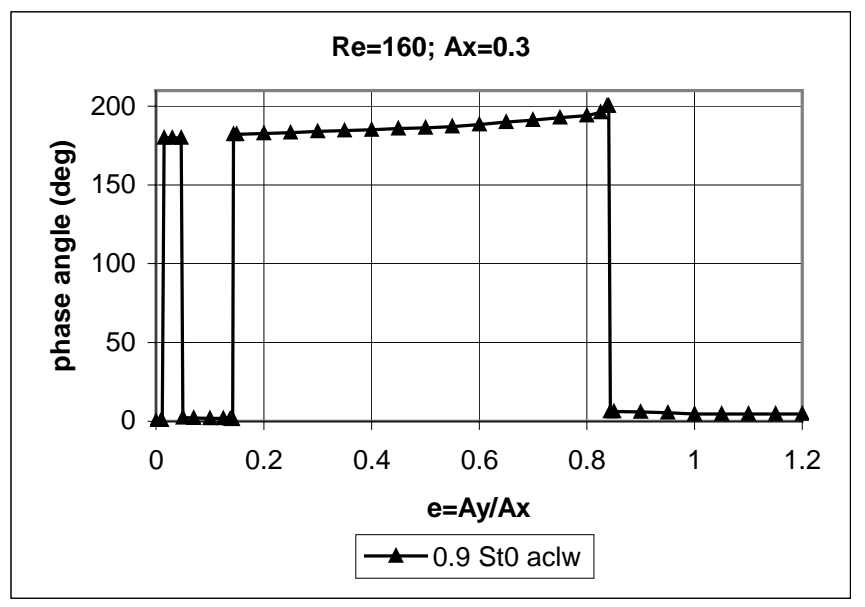

Figure 12 Phase angle vs. ellipticity

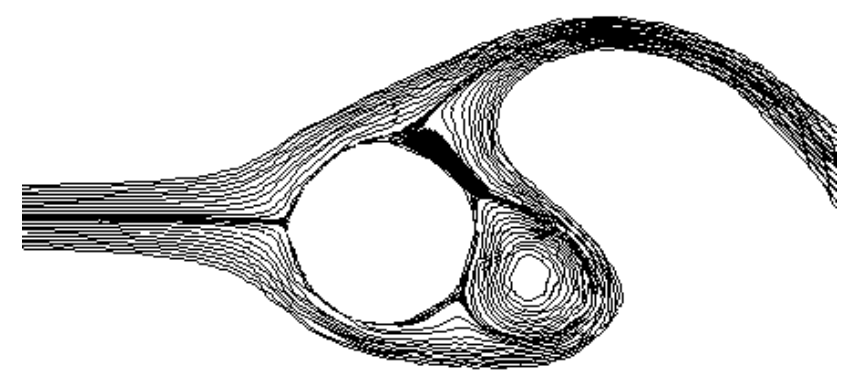

Figure 13a Streamlines for $A_{y}=\mathbf{0 . 0 4 2 9}$

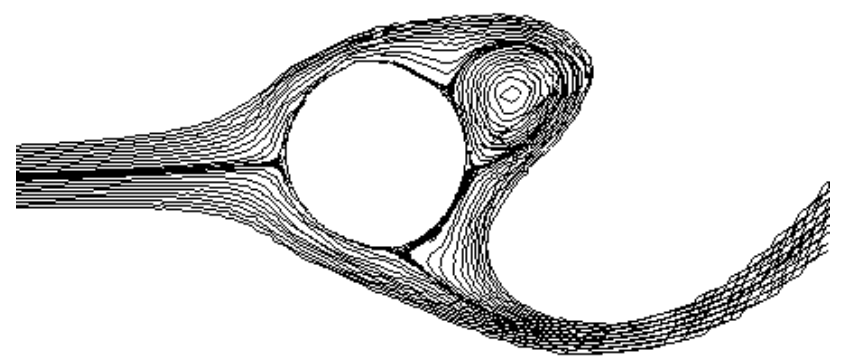

Figure 13b Streamlines for $A_{y}=\mathbf{0 . 0 4 3 2}$

\section{EFFECT OF ORBITAL DIRECTION}

Here, one set of results will be shown for the case of both clockwise and anticlockwise orbits at $R e=160, A_{x}=0.3$, and $f_{x}=f_{y}=0.9 S t_{0}=0.16938$ where $S t_{0}$ is the Strouhal number for a stationary cylinder at $R e=160$. This set was representative of the several sets of computations performed.

Figure 14 gives the TMV of $C_{L}$ versus ellipticity $e$. The filled triangles show results for a cylinder orbiting anticlockwise (aclw in the figure), similar to Fig. 3. Note that again there are two envelope curves, which are roughly parallel to each other and of negative slope. On the other hand, the empty squares in Fig. 14 show results for a clockwise (clw) orbit, with the other parameters unchanged. The two envelope curves can be seen, again roughly parallel, but the slope is positive, and they are a mirror image of the envelope curves of the cylinder orbiting anticlockwise. There are four jumps or switches in state, although this is difficult to identify at this scale. In all calculations made so far, $\bar{C}_{L}$ has shown this pattern: a mirror image of each other, with the slope of the anticlockwise curve being negative and the clockwise positive.

The rest of the TMV and rms values plotted against ellipticity shown in Figs. 15-19 are basically unaffected by the direction of orbit. Results belonging to the clockwise and anticlockwise direction of orbit fall on the same envelope curves and even the location of jumps coincide in all cases. It is difficult to distinguish the two curves. The other TMV and rms values are shown, although unlike the TMV of lift they are not affected by the direction of orbit, in order to give a full display of the effect of ellipticy upon these values.

Figure 15 shows the rms value of the lift coefficient. Because the curves belonging to the anticlockwise and clockwise orbits coincide for the most part, the two curves are difficult to distinguish, but clearly they are almost identical. As in Fig. 4, the two envelope curves intersect at 0 ellipticity. Here, in contrast with Fig. 14, the two pairs of envelope curves belonging to the two orbital directions coincide. The locations of the jumps are at the same $e$ values as for $\bar{C}_{L}$.

Figures 16 and 17 give the TMV of the drag and base pressure coefficients versus ellipticity $e$. As in Fig. 15, the two pairs of envelope curves intersect each other at $e=0$ and they coincide. Figures 18 and 19 show the rms of the drag and base pressure coefficient, with similar results. 


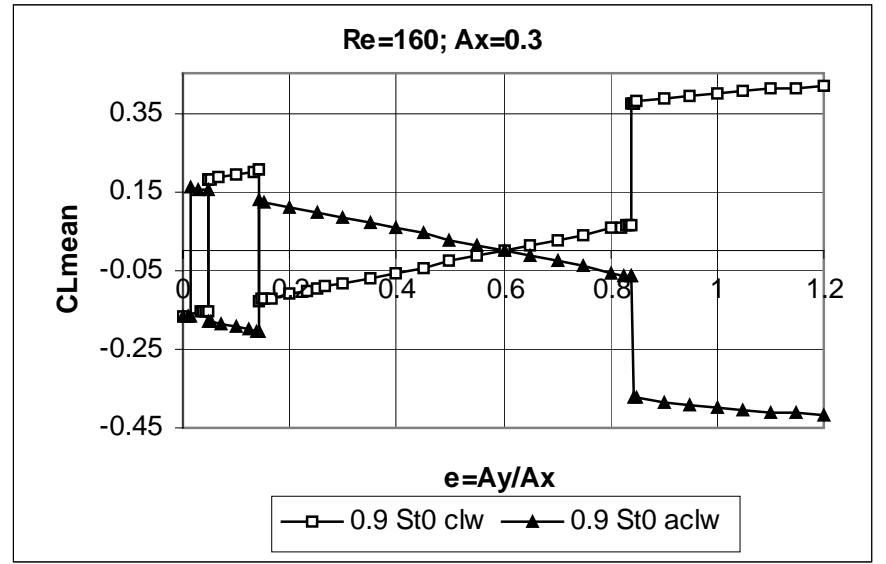

Figure 14 Time-mean value of lift vs. ellipticity

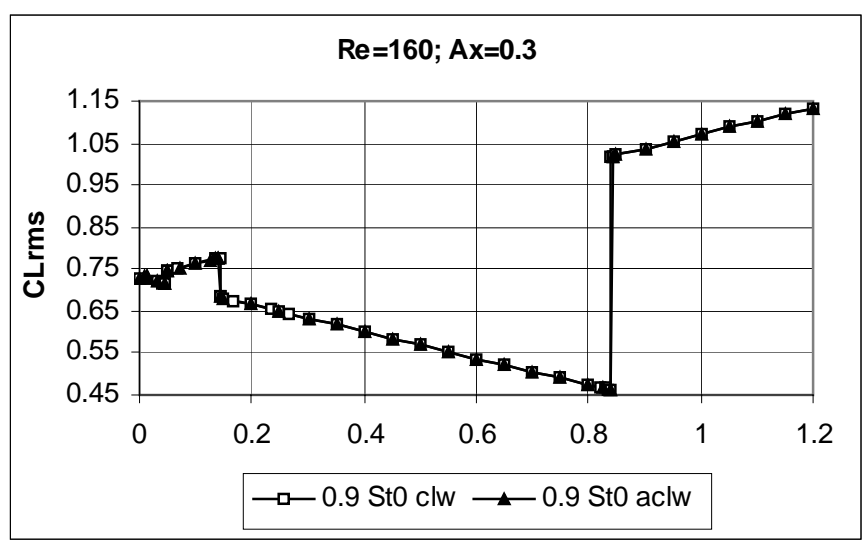

Figure 15 Root-mean square value of lift vs. ellipticity

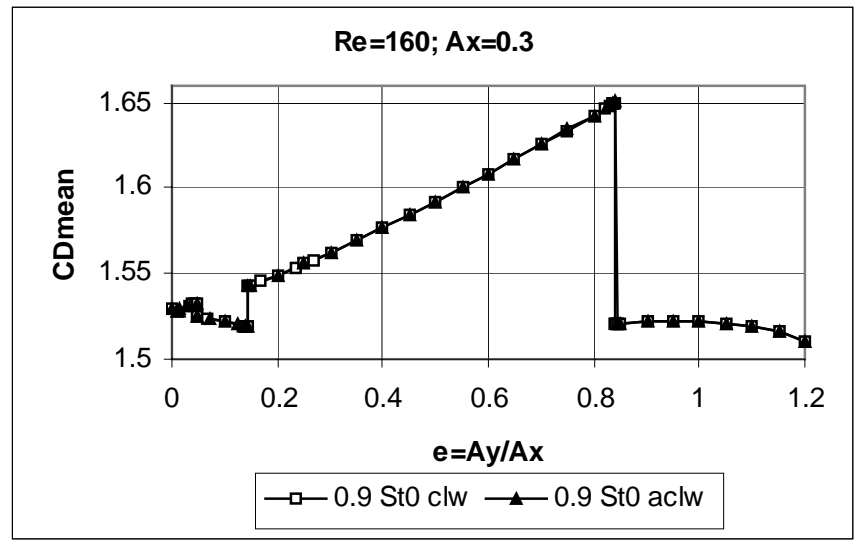

Figure 16 Time-mean value of drag vs. ellipticity

From the sets of computations for the two orbital directions, it is clear that two pairs of envelope or state curves exist that in most cases coincide with each other. This is reassuring in two ways: (1) The code produces the same timemean and rms results for two different situations represented by the two directions of orbit, and this confirms that the code is reliable, and (2) the existence of envelope curves is proved by results obtained for two different cases

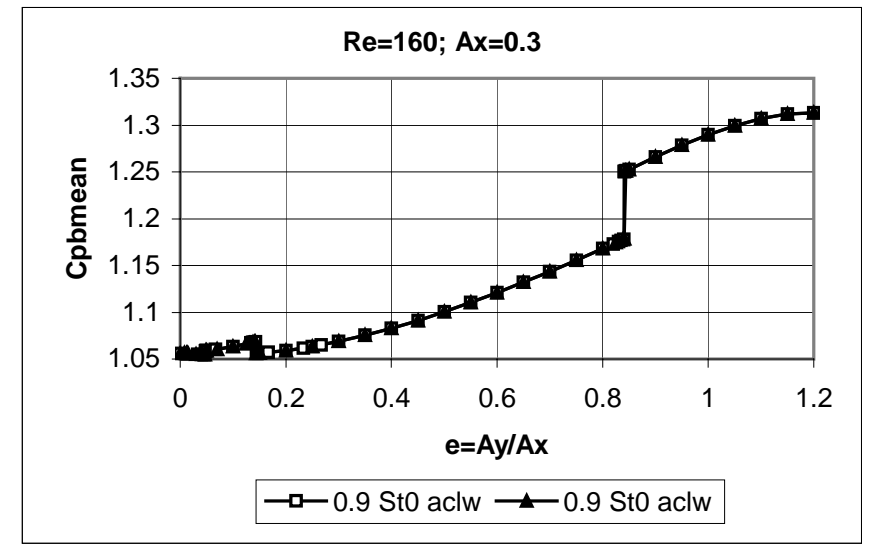

Figure 17 Time-mean value of base pressure vs. ellipticity

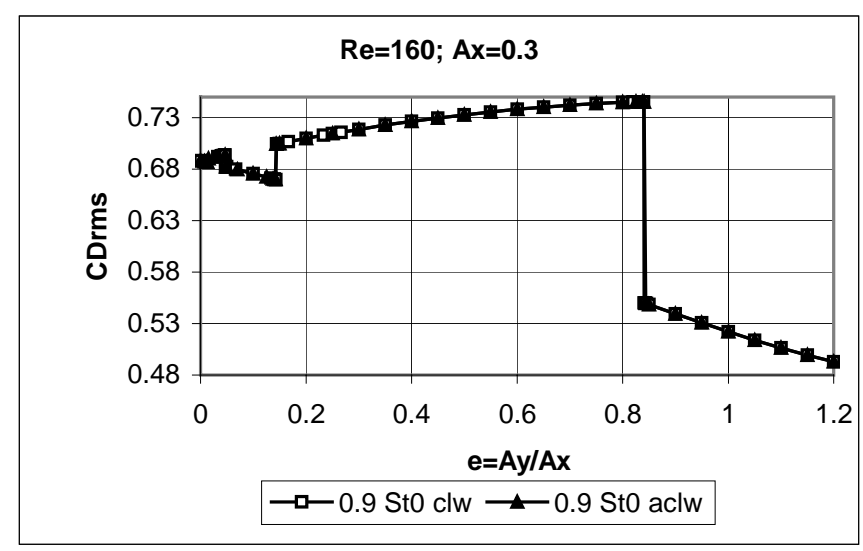

Figure 18 Root-mean square value of drag vs. ellipticity

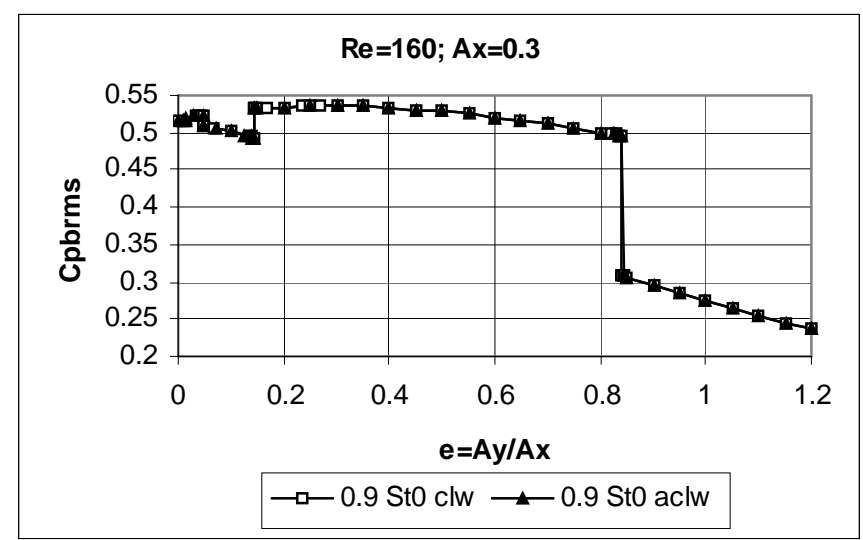

Figure 19 Root-mean square value of base pressure vs. ellipticity

Apart from TMV of lift, it was found that the direction of orbit has basically no effect on TMV and rms values of force coefficients. However, it may be worth noting that since $\bar{C}_{L}$ is affected, it may be necessary to take the direction of orbit into consideration when using results from orbital studies, most of which are done with only one orbital direction. 


\section{EFFECT OF INITIAL CONDITION}

For earlier clockwise computations, the initial conditions were $x_{0}(t=0)=A_{x}, y_{0}(t=0)=0$ (3 o'clock position). The computations were repeated for a clockwise orbit using a different initial condition in which $x_{0}(t=0)=-A_{x}$, $y_{0}(t=0)=0$ (9 o'clock position). For all cases, including $\bar{C}_{L}$ (shown in Fig. 20; in contrast with Fig. 14), the two pairs of envelope curves coincided, but the location of jumps was somewhat affected by the initial conditions (cf. Figs. 21 and 5). In Figs. 20 and 21, the envelope curves marked with the open squares are the same as those shown in Fig. 5; the filled diamonds refer to the changed initial conditions (clwic). A comparison shows that the jumps are at different $e$ values than before. All of the other data show the same tendency.

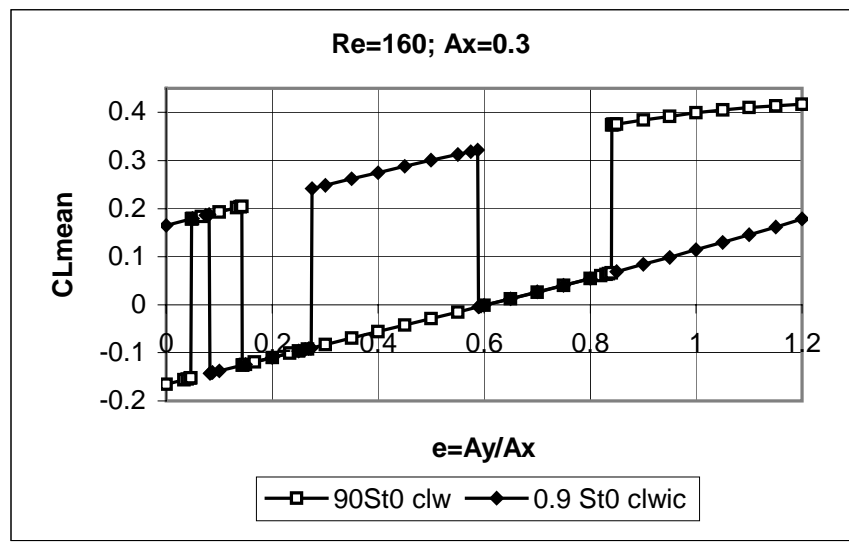

Figure 20 Time-mean value of lift vs. ellipticity, effect of initial condition

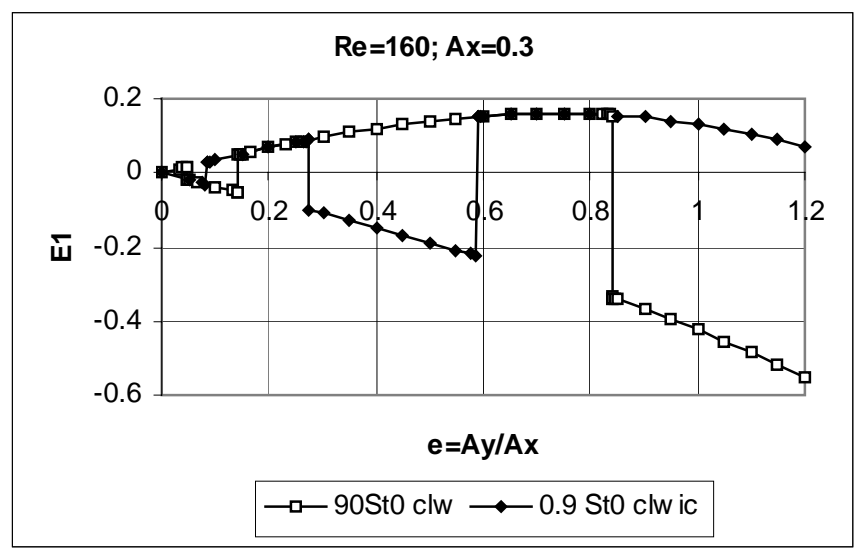

Figure 21 Transverse energy transfer $E_{1}$ vs. ellipticity, effect of initial condition

It is interesting that although the envelope curves are identical, the location of the jumps have been changed by shifting the starting point of calculations. For all five sets of computations at different $R e$ and $A_{x}$, specified at the beginning of this section, exactly the same type of results were obtained. For each set of variables (the TMV and rms of $C_{L}, C_{D}$ and $C_{p b}$, and the mechanical energy transfer $E_{1}, E_{2}$ and $E$ ), the location of jumps for the same initial condition was always the same. Although this effect cannot be explained at this point, the consistent results indicate that it is not a fluke, and is worth examining further.

\section{CONCLUSIONS}

The definition of mechanical energy transfer between a transversely oscillated cylinder and fluid [3] was extended here for use with a cylinder mechanically forced to follow an orbital path. Energy transfer in this case is composed of two parts, $E_{1}$ for the energy transfer in the transverse direction, and $E_{2}$ for the in-line direction. These variables were investigated against different values of ellipticity. Two envelope curves were detected, between which the solution abruptly jumped. For $E_{1}$, values were both positive and negative, while $E_{2}$ was always negative, as was the sum of the two. Limit cycle curves were investigated prior to and immediately after a jump, and those for lift and transverse cylinder displacement showed a sign switch, while those for drag and in-line displacement were hardly affected around the jump. Flow patterns for the two ellipticity values around the jump were plotted at the same phase in the cylinder motion cycle, and illustrate the drastic effect on the timing of vortex shedding of a very small change in ellipticity value at a jump.

The phase angle between lift and transverse displacement $\Phi_{L}$ switched by about $180^{\circ}$ at the jump, while no change was detected for the phase angle between drag and in-line displacement. When $\Phi_{L}$ was determined for the entire range of ellipticity investigated, this approximately $180^{\circ}$ change was observed at every jump.

The effect of the direction of orbit was important only in the case of the time-mean value of the lift coefficient. An alteration of $180^{\circ}$ in phase angle in the initial conditions left the envelope curves unaffected but somewhat modified the location of jumps.

These results serve to support the evidence for a physical phenomenon resulting in sudden jumps at low Reynolds numbers in the case of an orbiting cylinder. The evidence now includes changes in time-history of lift, limit cycles, mechanical energy transfer, phase angle, and flow patterns.

\section{ACKNOWLEDGMENTS}

The support provided by the Hungarian Research Foundation (OTKA, Project No. T 042961) is gratefully acknowledged. The author also thanks Mr. S. Ujvárosi for preparing Fig. 13.

\section{REFERENCES}

[1] Bearman, P. W., Downie, M.J., Graham, J. M. R. and Obasaju, E. D., 1985, "Forces on cylinders in viscous oscillatory flow at low Keulegan-Carpenter numbers," Journal of Fluid Mechanics 154, pp. 337-356.

[2] Sarpkaya, T., 1986, "Force on a circular cylinder in viscous oscillatory flow at low Keulegan-Carpenter numbers,” Journal of Fluid Mechanics 165, pp. 61-71. 
[3] Blackburn, H.M. and Henderson. R.D., 1999, “A study of two-dimensional flow past an oscillating cylinder." Journal of Fluid Mechanics 385, pp. 255-286.

[4] Meneghini, J. R. and Bearman, P. W., 1995, "Numerical simulation of high amplitude oscillatory flow about a circular cylinder,” Journal of Fluids and Structures 9, pp. 435-455.

[5] Williamson, C. H. K. and Roshko, A., 1988, "Vortex formation in the wake of an oscillating cylinder," Journal of Fluids and Structures 2, pp. 355-381.

[6] Teschauer, I., Schäfer, M. and Kempf, A., 2002, "Numerical simulation of flow induced by a cylinder orbiting in a large vessel," Journal of Fluids and Structures 16(4), pp. 435-451.

[7] Stansby, P.K. and Rainey, R.C.T., 2001, "On the orbital response of a rotating cylinder in a current," Journal of Fluid Mechanics 439, pp. 87-108.

[8] Blackburn, H. M. and Karniadakis, G.E., 1993, “Two-and Three-Dimensional Simulations of Vortex-Induced Vibration of a Circular Cylinder," Paper presented at the $3^{\text {rd }}$ Int. Offshore \& Polar Engineering Conference, Singapore.

[9] Lu, X.Y. and Dalton, C., 1996, "Calculation of the timing of vortex formation from an oscillating cylinder," Journal of Fluids and Structures 10, pp. 527-541.

[10]Baranyi, L., 2003, "Computation of unsteady momentum and heat transfer from a fixed circular cylinder in laminar flow," Journal of Computational and Applied Mechanics 4(1), pp. 13-25.

[11] Kawamura, T. and Kuwahara, K., 1984, "Computation of high Reynolds number flow around a circular cylinder with surface roughness", Proc. $22^{\text {nd }}$ Aerospace Sci. Meeting, Reno, Nevada, AIAA-84-0340, pp. 1-11.

[12] Poncet, P., 2002, "Vanishing of B mode in the wake behind a rotationally oscillating cylinder," Physics of Fluids 14(6), pp. 2021-2023.

[13] Barkley, D. and Henderson, R. D., 1996, "Threedimensional Floquet stability analysis of the wake of a circular cylinder," Journal of Fluid Mechanics 322, pp. 215-241.

[14] Baranyi, L., 2005, “Abrupt changes in the root-meansquare values of force coefficients for an orbiting cylinder in uniform stream," Proceedings of the $4^{\text {th }}$ Symposium on Bluff Body Wakes and Vortex-Induced Vibrations, Santorini, Greece, pp. 55-58.

[15] Baranyi, L., 2004, "Numerical simulation of flow past a cylinder in orbital motion”, Journal of Computational and Applied Mechanics 5(2), pp. 209-222.

[16] Blackburn, H.M., 2003, “Computational bluff body fluid dynamics and aeroelasticity," in Barton, N.G. and Periaux, J. (Eds), Coupling of Fluids, Structures and Waves Problems in Aeronautics, Notes on Numerical Fluid Mechanics and Multidisciplinary Design (NNFM), Vol. 85, Springer, pp. 10-23. 